Supporting Information for

\title{
Quantitative Analysis and Anti-inflammatory Activity Evaluation of the A-Type Avenanthramides in Commercial Sprouted Oat
}

\section{Products}

\section{Changling $\mathrm{Hu}^{\dagger}$, Yao Tang ${ }^{\dagger}$, Yantao Zhao, Shengmin Sang*}

Laboratory for Functional Foods and Human Health, Center for Excellence in Post-Harvest Technologies, North Carolina Agricultural and Technical State University, North Carolina Research Campus, 500 Laureate Way, Kannapolis, North Carolina 28081, United States

†These authors contributed equally to this study.

\section{Corresponding authors:}

Shengmin Sang, Ph.D.

North Carolina Agricultural and Technical State University

North Carolina Research Campus

Tel: 704-250-5710; Fax: 704-250-5709

Email: ssang@ncat.edu or shengminsang@yahoo.com 


\section{Contents}

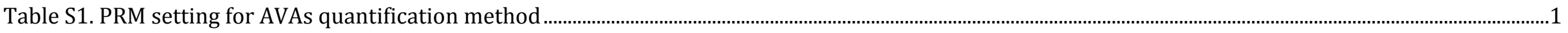

Table S2. Contents of C-type AVAs in Seven Commercial Oat Products ( $\mu \mathrm{g} / \mathrm{g}$ of products) ……………………...............................................................................

Figure S1. HPLC-DAD response of 2p, 2c, 2f, 2 $\mathrm{p}_{\mathrm{d}}, 2 \mathrm{c}_{\mathrm{d}}$ and $2 \mathrm{f}_{\mathrm{d}}(340 \mathrm{~nm}, \mathrm{c}=10 \mu \mathrm{M}$ for each standard).........................................................................................

Figure S2. MS Fragments of seven purified A-type AVAs 
Table S1. PRM setting for AVAs quantification method

\begin{tabular}{|c|c|c|c|c|c|c|c|c|}
\hline Mass $[\mathrm{m} / \mathrm{z}]$ & Formula [M] & Formula type & Species & $\mathrm{CS}[\mathrm{z}]$ & Polarity & Start [min] & End [min] & NCE $(\%)$ \\
\hline 476.1198 & $\mathrm{C} 22 \mathrm{H} 23 \mathrm{NO} 11$ & Chemical formula & 2c-glu & 1 & Negative & 3.6 & 4.85 & 40 \\
\hline 330.0619 & C16H13N O7 & Chemical formula & $5 c$ & 1 & Negative & 4.4 & 5.3 & 40 \\
\hline 314.0670 & C16H13N O6 & Chemical formula & $2 c, 4 c, 5 p$ & 1 & Negative & 5.25 & 6.6 & 40 \\
\hline 344.0776 & $\mathrm{C} 17 \mathrm{H} 15 \mathrm{~N} \mathrm{O} 7$ & Chemical formula & $5 \mathrm{f}$ & 1 & Negative & 5.5 & 6.5 & 40 \\
\hline 356.0776 & $\mathrm{C} 18 \mathrm{H} 15 \mathrm{NO} 7$ & Chemical formula & $5 c_{d}$ & 1 & Negative & 5.6 & 6.6 & 40 \\
\hline 298.0721 & C16H13N O5 & Chemical formula & $2 \mathrm{p}, 4 \mathrm{p}, 1 \mathrm{c}$ & 1 & Negative & 5.9 & 7.6 & 40 \\
\hline 358.0932 & C18H17NO7 & Chemical formula & $3 f$ & 1 & Negative & 6.15 & 7.4 & 40 \\
\hline 328.0827 & C17H15N O6 & Chemical formula & $2 f, 4 f, 3 p$ & 1 & Negative & 6.2 & 7.2 & 40 \\
\hline 340.0827 & C18H15N O6 & Chemical formula & $2 \mathrm{c}_{\mathrm{d}}, 4 \mathrm{c}_{\mathrm{d}}$ & 1 & Negative & 6.3 & 7.3 & 40 \\
\hline 370.0932 & C19H17NO7 & Chemical formula & $5 \mathrm{f}_{\mathrm{d}}$ & 1 & Negative & 6.5 & 7.4 & 40 \\
\hline 324.0878 & C18H15N O5 & Chemical formula & $2 \mathrm{p}_{\mathrm{d}}, 4 \mathrm{p}_{\mathrm{d}}, 1 \mathrm{c}_{\mathrm{d}}$ & 1 & Negative & 6.95 & 8.4 & 40 \\
\hline 354.0983 & C19H17N O6 & Chemical formula & $2 \mathrm{f}_{\mathrm{d}}, 4 \mathrm{f}_{\mathrm{d}}, 3 \mathrm{p}_{\mathrm{d}}$ & 1 & Negative & 7.2 & 8.2 & 40 \\
\hline 384.1089 & C20H19N O7 & Chemical formula & $3 \mathrm{f}_{\mathrm{d}}$ & 1 & Negative & 7.45 & 7.95 & 40 \\
\hline 282.0772 & C16H13N O4 & Chemical formula & $1 \mathrm{p}$ & 1 & Negative & 7.5 & 9 & 40 \\
\hline 312.0878 & C17H15N O5 & Chemical formula & 1f & 1 & Negative & 7.8 & 8.7 & 40 \\
\hline 326.1034 & C18H17NO5 & Chemical formula & Internal standard & 1 & Negative & 8 & 9.5 & 40 \\
\hline 308.0928 & C18H15N O4 & Chemical formula & $1 p_{d}$ & 1 & Negative & 8.25 & 9.15 & 40 \\
\hline 338.1034 & C19H17N O5 & Chemical formula & $1 f_{d}$ & 1 & Negative & 8.35 & 9.2 & 40 \\
\hline 344.0776 & C17H15N O7 & Chemical formula & $5 f$ & 1 & Negative & 9.55 & 10.85 & 40 \\
\hline
\end{tabular}


Table S2. Contents of C-type AVAs in Seven Commercial Oat Products ( $\mu \mathrm{g} / \mathrm{g}$ of products) ${ }^{a, b}$

\begin{tabular}{|c|c|c|c|c|c|c|c|c|c|c|c|c|c|c|c|c|}
\hline No & $1 c$ & $2 c$ & $4 c$ & $5 c$ & $1 p$ & $2 p$ & $3 p$ & $4 p$ & $5 p$ & 1f & $2 f$ & $3 f$ & $4 f$ & $5 f$ & 2c-glu & $\begin{array}{l}\text { Subtotal } \\
(\mu \mathrm{g} / \mathrm{g})\end{array}$ \\
\hline \multirow[t]{2}{*}{1} & 13.44 & $81.20 \pm$ & $1.06 \pm$ & $1.24 \pm 0$ & $3.61 \pm 0$ & $121.70 \pm$ & $0.66 \pm 0$ & $1.50 \pm 0$ & $3.4 \pm 0$ & $0.67 \pm 0$ & $46.60 \pm$ & $19.60 \pm$ & $0.94 \pm 0$ & $2.29 \pm 0$ & $13.23 \pm$ & $310.95 \pm 2.9$ \\
\hline & \pm 0.30 & 160 & & .02 & .05 & 1.60 & .02 & .02 & 07 & .03 & & 0.59 & .06 & .03 & 0.16 & 3 \\
\hline \multirow[t]{2}{*}{2} & $0.94 \pm$ & $10.69 \pm$ & $0.03 \pm$ & trace & $0.16 \pm 0$ & $14.10 \pm 0$ & $0.09 \pm 0$ & $0.16 \pm 0$ & $0.13 \pm 0$ & $0.07 \pm 0$ & $7.85 \pm$ & $2.64 \pm 0$ & $0.17 \pm 0$ & trace & $1.72 \pm 0$ & $38.80 \pm 0.97$ \\
\hline & 0.02 & 0.23 & & & .01 & 46 & .01 & .02 & .00 & .01 & & 14 & .02 & & 22 & \\
\hline \multirow[t]{2}{*}{3} & $1.16 \pm$ & $11.71 \pm$ & $0.06 \pm$ & trace & $0.13 \pm 0$ & $12.20 \pm 0$ & $0.08 \pm 0$ & $0.18 \pm 0$ & $0.12 \pm 0$ & $0.06 \pm 0$ & $7.36 \pm$ & $2.24 \pm 0$ & $0.11 \pm 0$ & trace & $2.05 \pm 0$ & $37.49 \pm 0.57$ \\
\hline & 0.05 & 0.15 & & & .01 & 11 & .02 & .01 & .00 & .01 & & 08 & .01 & & 08 & \\
\hline \multirow[t]{2}{*}{4} & $2.30 \pm$ & $20.36 \pm$ & $0.14 \pm$ & trace & $0.48 \pm 0$ & $25.29 \pm 1$ & $0.09 \pm 0$ & $0.28 \pm 0$ & $0.26 \pm 0$ & $0.10 \pm 0$ & $10.22 \pm$ & $2.01 \pm 0$ & $0.14 \pm 0$ & trace & $2.15 \pm 0$ & $63.96 \pm 2.51$ \\
\hline & 0.10 & 0.59 & & & .02 & 22 & .02 & .00 & .02 & .01 & & 02 & .01 & & 33 & \\
\hline \multirow[t]{2}{*}{5} & $2.09 \pm$ & $18.57 \pm$ & $0.12 \pm$ & trace & $0.43 \pm 0$ & $23.80 \pm 0$ & $0.10 \pm 0$ & $0.26 \pm 0$ & $0.22 \pm 0$ & $0.09 \pm 0$ & $9.19 \pm$ & $1.75 \pm 0$ & $0.13 \pm 0$ & trace & $2.69 \pm 0$ & $59.45 \pm 2.06$ \\
\hline & 0.07 & 0.67 & & & .02 & 88 & .01 & .02 & .01 & .00 & & 05 & .01 & & 24 & \\
\hline \multirow[t]{2}{*}{6} & $0.41 \pm$ & $6.97 \pm 0$ & $0.02 \pm 0$ & trace & trace & $4.86 \pm 0.1$ & & $0.11 \pm 0$ & $0.13 \pm 0$ & & $2.86 \pm 0$ & $0.49 \pm 0$ & $0.06 \pm 0$ & trace & $0.02 \pm 0$ & $15.93 \pm 0.50$ \\
\hline & 0.02 & 33 & .01 & & & 8 & & .00 & .00 & & .09 & 04 & .00 & & 01 & \\
\hline \multirow[t]{2}{*}{7} & $2.08 \pm$ & $18.41 \pm$ & $0.13 \pm$ & trace & $0.31 \pm 0$ & $21.94 \pm 1$ & $0.24 \pm 0$ & $0.42 \pm 0$ & $0.63 \pm 0$ & $0.10 \pm 0$ & $14.12 \pm$ & $5.08 \pm 0$ & $0.29 \pm 0$ & $0.31 \pm 0$ & $4.20 \pm 0$ & $69.65 \pm 4.15$ \\
\hline & 0.18 & 0.76 & & & .02 & 40 & .03 & .04 & .02 & .00 & & 28 & .02 & .03 & 57 & \\
\hline
\end{tabular}

a Values expressed as mean \pm standard deviation.

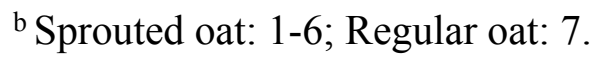


Figure S1. HPLC-DAD response of $2 \mathrm{p}, 2 \mathrm{c}, 2 \mathrm{f}, 2 \mathrm{p}_{\mathrm{d}}, 2 \mathrm{c}_{\mathrm{d}}$ and $2 \mathrm{f}_{\mathrm{d}}(340 \mathrm{~nm}, \mathrm{c}=10 \mu \mathrm{M}$ for each standard).

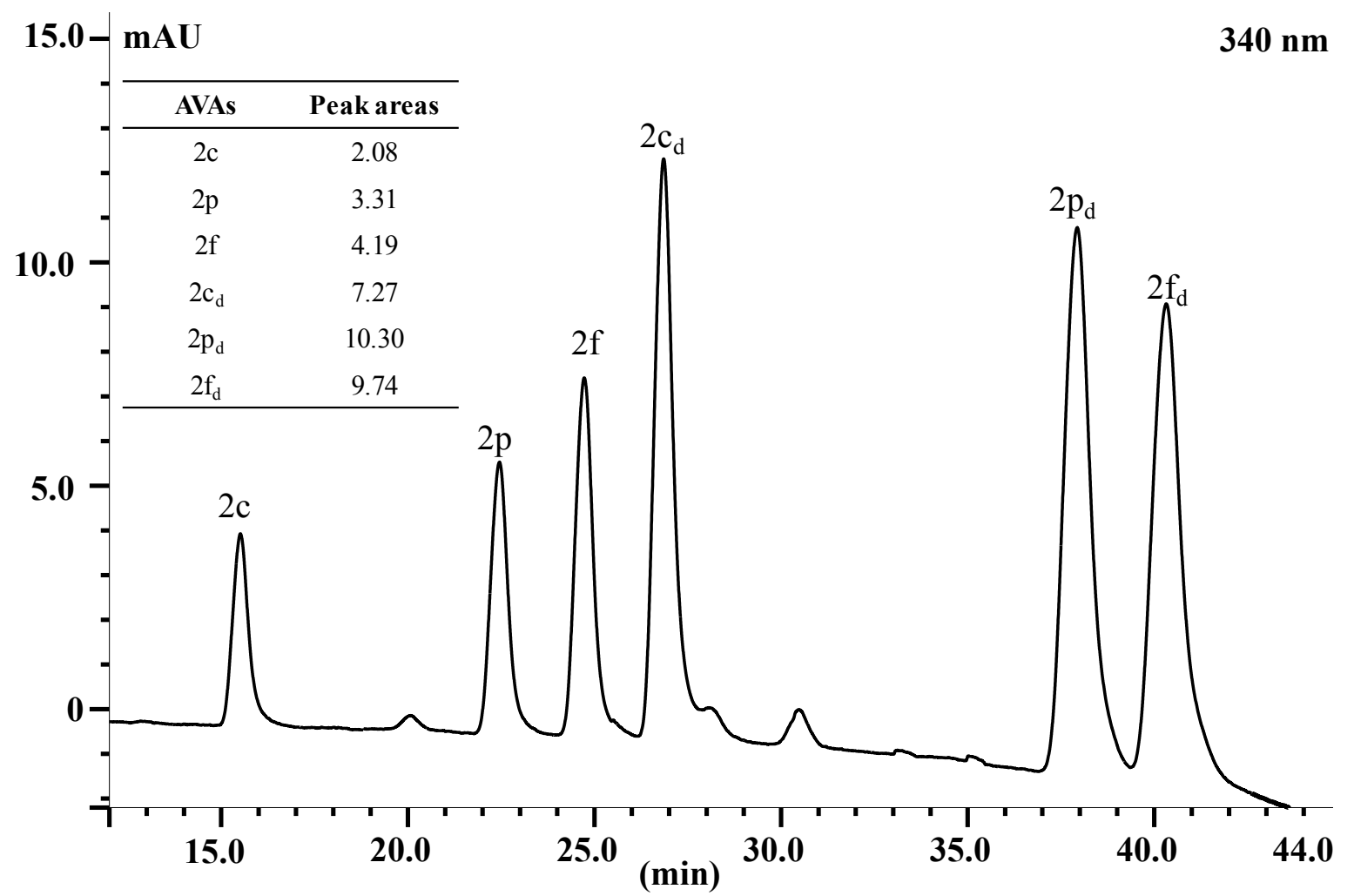

UHPLC analysis was performed on a Thermo LPG 3400 R.S system (Thermo Scientific, San Jose, CA). Column used was a gemini C18 column (150 × 4.6 mm, 5 $\mu \mathrm{m}$; Phenomenex). The mobile phase was consisted of water containing $0.1 \%$ formic acid (mobile phase A) and acetonitrile with $0.1 \%$ formic acid (mobile phase B). The gradient elution was carried out for $60 \mathrm{~min}$ at a flow rate of $1.0 \mathrm{~mL} / \mathrm{min}$. A gradient eluting system was applied as follow: 0-5 min, 20\% B; 5-25 min, 20-26\% B; $26-28 \mathrm{~min}, 25-48 \% \mathrm{~B} ; 48-50 \mathrm{~min}, 28-100 \% \mathrm{~B} ; 50-55 \mathrm{~min}, 100 \%$ B. The column was then re-equilibrated with $20 \%$ B from 56 to $60 \mathrm{~min}$. 
Figure S2. MS Fragments of seven purified A-type AVAs

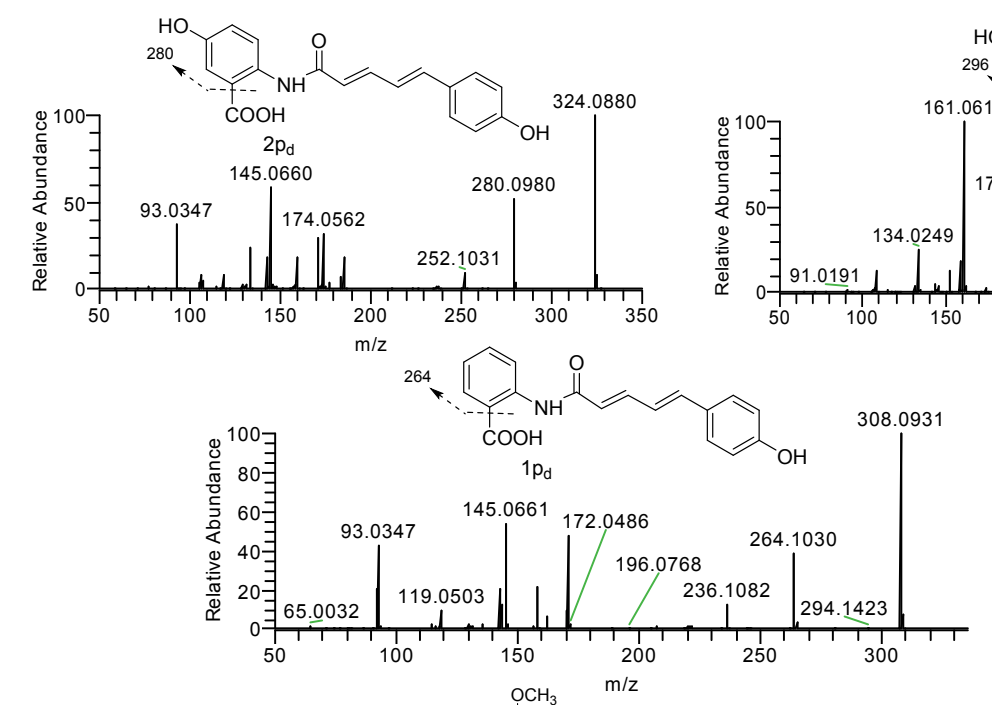

(178)

$\cdots \mathrm{NH}^{\mathrm{OH}}$

$\mathrm{COOH}^{2}$

187.0403

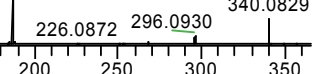

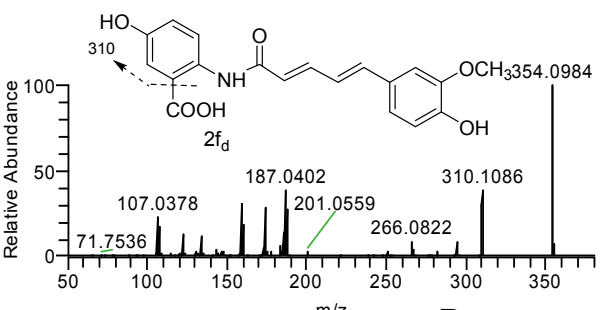

m

$\mathrm{R}_{2}$
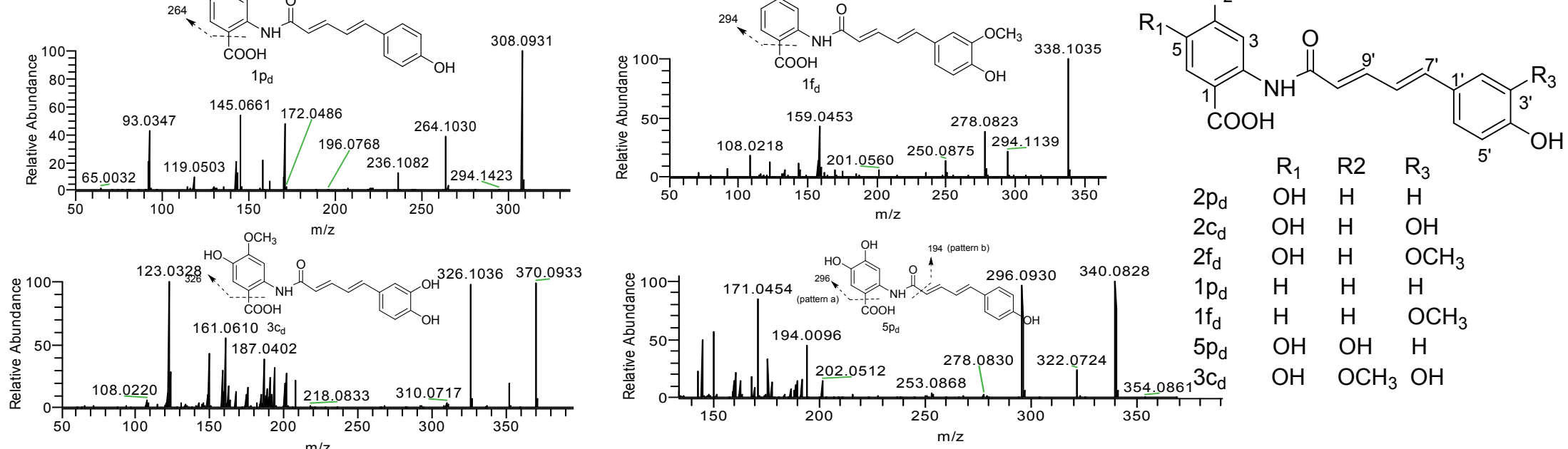

$\mathrm{R}_{1} \quad \mathrm{R} 2 \quad \mathrm{R}_{3}$

$2 \mathrm{p}_{\mathrm{d}} \mathrm{OH} \quad \mathrm{H} \quad \mathrm{H}$

$2 \mathrm{c}_{\mathrm{d}} \mathrm{OH} \quad \mathrm{H} \quad \mathrm{OH}$

$\begin{array}{llll}2 \mathrm{f}_{\mathrm{d}} & \mathrm{OH} & \mathrm{H} & \mathrm{OCH}_{3}\end{array}$

$\begin{array}{llll}\mathrm{p}_{\mathrm{d}} & \mathrm{H} & \mathrm{H} & \mathrm{H}\end{array}$

$\begin{array}{llll}\mathrm{f}_{\mathrm{d}} & \mathrm{H} & \mathrm{H} & \mathrm{OCH}_{3}\end{array}$

$\begin{array}{llll}5 p_{d} & \mathrm{OH} & \mathrm{OH} & \mathrm{H}\end{array}$

$\mathrm{OH} \quad \mathrm{OCH}_{3} \mathrm{OH}$ 